\title{
Xanthogranulomatous pituitary adenoma: A case report and literature review
}

\author{
GUIHONG LI ${ }^{1}$, CHAOCHAO ZHANG $^{1}$, YUXUE SUN ${ }^{1}$, QINGCHUN MU ${ }^{2}$ and HAIYAN HUANG ${ }^{1}$ \\ ${ }^{1}$ Department of Neurosurgery, The First Hospital of Jilin University, Changchun, Jilin 130021; \\ ${ }^{2}$ Department of Neurosurgery, Hongqi Hospital of Mudanjiang Medical University, \\ Mudanjiang, Heilongjiang 157011, P.R. China
}

Received September 21, 2016; Accepted November 17, 2017

DOI: $10.3892 / \operatorname{mco} .2018 .1547$

\begin{abstract}
Xanthogranuloma, also referred to as cholesterol granuloma or xanthogranulomatous reaction, is a granulomatous lesion that is infrequently found in the sellar and parasellar regions. Xanthogranulomatous pituitary adenoma is relatively rare and, thus, the etiology, diagnosis, management and prognosis of this condition remain incompletely understood. We herein report the case of a 56-year-old female patient who presented to our institution with intermittent headache, vomiting and distending pain in the bilateral orbital regions. Brain magnetic resonance imaging revealed a sellar mass with a heterogeneous signal. The mass was subtotally resected, and histopathological examination confirmed the diagnosis of xanthogranulomatous pituitary adenoma. Although the patient's symptoms were relieved following surgical treatment, intractable hyponatremia and diabetes insipidus developed and she received hormone replacement therapy. At the last follow-up (November 2016), the patient remained recurrence-free. A total of 14 cases of pituitary adenoma with concomitant xanthogranuloma were identified in the literature, and the clinical and radiological manifestations are discussed. Sellar xanthogranuloma is usually associated with craniopharyngioma or Rathke's cleft cyst; however, it may also occur in isolation. Xanthogranulomatous pituitary adenomas are infrequent, making their diagnosis challenging. Surgical resection is the preferred treatment, and attention should be paid to postoperative hypopituitarism and development of diabetes insipidus.
\end{abstract}

Correspondence to: Professor Haiyan Huang, Department of Neurosurgery, First Hospital of Jilin University, 71 Xinmin Avenue, Changchun, Jilin 130021, P.R. China

E-mail: huanghaiyanns@hotmail.com

Dr Qingchun Mu, Department of Neurosurgery, Hongqi Hospital of Mudanjiang Medical University, Mudanjiang, Heilongjiang 157011, P.R. China

E-mail: 1527032637@qq.com

Key words: pituitary adenoma, xanthogranuloma, sellar region, diabetes insipidus

\section{Introduction}

Sellar xanthogranuloma, also referred to as cholesterol granuloma or xanthogranulomatous reaction, is a rare granulomatous lesion consisting of cholesterol clefts, hemosiderin deposits, macrophages, chronic inflammatory infiltrates and fibrous proliferation $(1,2)$. Sellar xanthogranuloma is most commonly associated with craniopharyngioma or Rathke's cleft cyst, but may also occur in isolation (2). The xanthogranulomatous change was first described in 1988 (3). Xanthogranulomatous pituitary adenomas are extremely rare, with only a few cases reported in the literature to date $(1,3,4)$. Therefore, the etiology, diagnosis, management and prognosis of this condition have yet to be fully elucidated. We herein report a case of pituitary adenoma with concomitant xanthogranuloma in a female patient who developed diabetes insipidus postoperatively. The relevant literature is also reviewed and discussed.

\section{Case report}

A 56-year-old woman presented to The First Hospital of Jilin University (Changchun, China) on August 30, 2014, with a 20-day history of intermittent headache, vomiting and distending pain in the bilateral orbital regions. Brain computed tomography (CT) scanning revealed a sellar mass exhibiting heterogeneous hyperintensity (Fig. 1A). Subsequently, brain magnetic resonance imaging (MRI) revealed an intra- and suprasellar oval mass sized $30 \times 25 \times 30 \mathrm{~mm}$, involving the hypothalamus and the foramen of Monro. The pituitary gland and the pituitary stalk could not be clearly identified. The mass was heterogeneously isointense on T1-weighted images (WI) and heterogeneously hyperintense on T2WI, with significant enhancement following contrast administration (Fig. 1B and D). The third ventricle was deformed due to compression, and lateral ventricular dilation was observed. The laboratory examinations revealed a decreased serum cortisol hormone level at 8 a.m. (69.25 nmol/1; normal range, 240-619 nmol/1), with normal levels of other endocrine hormones: Luteinizing hormone (LH; $39.08 \mathrm{mIU} / \mathrm{ml}$; normal range, 10.87-58.64 $\mathrm{mIU} / \mathrm{ml}$ ), prolactin (PRL; $191.3 \mathrm{mIU} / \mathrm{l}$; normal range, 70.81-566.5 mIU/l) and growth hormone $(\mathrm{GH}$; $0.206 \mathrm{ng} / \mathrm{ml}$; normal range, 0.01-3.607 ng/ml). Preoperatively, the suspected diagnosis was pituitary adenoma. 
A craniotomy was performed 8 days after admission, via the right pterional approach. Intraoperatively, a soft pinkish-grey mass was identified, which was well-demarcated and hypervascular. The bilateral optic nerves were surrounded by the tumor and, after the optic nerve was isolated, the tumor was partially resected in a piecemeal fashion due to poor exposure. Cholesterol clefts were noted in the tumor. The posterior section of the tumor infiltrated the third ventricle, which could not be completely exposed, and gross total resection was not forced. The pituitary stalk, bilateral optic nerves and internal carotid artery were well-preserved.

Histopathological examination confirmed the diagnosis of plurihormonal xanthogranulomatous pituitary adenoma (Fig. 2A). In the xanthogranulomatous sections, cholesterol clefts, hemosiderin deposits, macrophages, chronic inflammatory infiltrates and fibrous proliferation were observed. Immunohistochemical staining revealed that the tumor was positive for synaptophysin (Syn), chromogranin A (CgA), GH, PRL, LH and thyroid-stimulating hormone (TSH) (Fig. 2B and C). The percentage of Ki-67-positive tumor cells was $\sim 1 \%$.

Postoperative MRI confirmed partial resection, with residual tumor noted above the optic chiasma, which remained heterogeneously isointense on T1WI and heterogeneously hyperintense on T2WI, with significant contrast enhancement (Fig. 3). Postoperative endocrine examinations revealed decreased serum levels of thyroid-related hormones (TSH=0.08 $\mu \mathrm{IU} / \mathrm{ml}$, normal range: $0.27-4.2 \mu \mathrm{IU} / \mathrm{ml}$; free triiodothyronine $=2.19 \mathrm{pmol} / 1$, normal range: $3.1-6.8 \mathrm{pmol} / \mathrm{l}$; and free thyroxine $=11.29 \mathrm{pmol} / 1$, normal range: $12.0-22.0 \mathrm{pmol} / \mathrm{l}$ ), cortisol (69.25 nmol/1; normal range: $240-619 \mathrm{nmol} / \mathrm{l})$ and adrenocorticotrophic hormone (ACTH; $0.72 \mathrm{pmol} / 1$; normal range: $1.6-13.9 \mathrm{pmol} / \mathrm{l})$. The $24-\mathrm{h}$ urine volume was 9.221 . Hypopituitarism was diagnosed and the patient was treated with hormone replacement.

At the 3-month follow-up after surgery, the patient's previous symptoms, including headache and vomiting, had completely resolved, and her vision and visual fields were normal. However, intractable hyponatremia and diabetes insipidus due to hypopituitarism remained. No progression of the residual tumor was observed on follow-up MRI. The last follow-up was performed in November 30, 2016, and the patient remained on hormone replacement therapy and recurrence-free. Written informed consent was obtained from the patient regarding the publication of the case details and accompanying images.

\section{Discussion}

A search the relevant literature yielded only 15 reported cases of pituitary adenoma with xanthogranulomatous change $(1,3,4)$. The clinical profiles of the 15 patients are summarized as follows. With respect to demographic characteristics, the male:female ratio was $0.2: 1$, with an apparent female predominance. The average patient age was 53 years (range, 33-67 years) and the tumors were located in the intra-, supra-, or parasellar regions. The clinical manifestations included hypopituitarism, visual impairment, headache and vomiting. Radiologically, the tumors were usually cystic, with heterogeneous intensity on MRI. The tumors appeared iso- to hyperintense on T1WI, and the intensity varied from hypo- to hyperintense on T2WI. Following gadolinium-DTPA (diethylenetriaminepentaacetic acid) administration, enhancement was usually significant and heterogeneous. The differential diagnosis included craniopharyngioma and Rathke's cleft cyst.

Surgical resection was performed in all 15 patients. In each case, the tumor was removed via the transsphenoidal approach. Gross total resection was achieved in 11 of the 15 cases (73.3\%). Intraoperatively, the tumors were usually soft, pinkish-grey and well-demarcated. Oily yellow liquid and fibrous tissue were found within the tumors. Pathologically, pituitary adenoma cells and xanthogranulomatous changes were observed, including cholesterol clefts, hemosiderin deposits, macrophages, chronic inflammatory infiltrates and fibrous proliferation. Immunohistochemical staining showed positivity for Syn, CgA, ACTH, GH, PRL, LH and TSH. In all 15 cases, the diagnosis was plurihormonal pituitary adenoma.

Symptoms of headache and visual impairment may be immediately relieved by surgical treatment; however, endocrine abnormalities may be difficult to resolve.

Sellar xanthogranuloma is a rare granulomatous lesion, which was first described by Shirataki et al in 1988 (3). Then in 1999, Paulus et al suggested that sellar xanthogranuloma is clinically and pathologically distinct from the classical adamantinomatous craniopharyngioma (2). According to a study published in 2011, the incidence of intracranial xanthogranuloma is $1.6-7 \%$, and lesions are only rarely found in the sellar and parasellar regions (5). Of the 643 patients with sellar or parasellar tumors retrospectively reviewed by Rahmani et al only 4 patients $(0.6 \%)$ had histologically confirmed xanthogranulomas (6). Additionally, in our review of the literature regarding sellar or parasellar xanthogranulomas, 160 cases were identified. Among those, 67 were pathologically diagnosed as isolated xanthogranulomas (41.8\%) (7-11), 52 (32.5\%) as xanthogranulomatous craniopharyngiomas $(2,3,6), 20$ $(12.5 \%)$ as xanthogranulomatous Rathke's cleft cysts $(5,6,12)$, $15(9.4 \%)$ as xanthogranulomatous pituitary adenomas $(1,3,4)$ and 6 cases $(3.8 \%)$ as xanthogranulomatous hypophysitis (13-15). To the best of our knowledge, the 15 cases of pituitary adenomas with xanthogranuloma are the only cases reported in the literature to date, confirming the rarity of this condition.

The etiology, the pathogenesis of sellar xanthogranulomas remains incompletely understood. One study proposed that xanthogranulomas may develop secondary to hemorrhage, inflammation, or degeneration (6). Amano et al analyzed the pathological characteristics of 7 cases of sellar xanthogranuloma and observed components of Rathke's cleft cyst in 6/7 cases (12); thus, they considered sellar xanthogranuloma as a terminal condition resulting from a secondary reaction caused by repeated inflammation, hemorrhage and degeneration of a Rathke's cleft cyst. Other studies proposed that xanthogranulomas may occur as a component of systemic autoimmune disease; secondary to a reactive degenerative response to an epithelial lesion such as craniopharyngioma, Rathke's cleft cyst, or pituitary adenoma; or within the context of multiorgan involvement related to conditions such as tuberculosis, sarcoidosis, or Erdheim-Chester disease (16-18).

The most common symptoms of xanthogranulomatous pituitary adenomas include vision and visual field disorders, hypopituitarism and headache. Nishioka et al analyzed the endocrinological and radiological characteristics of 

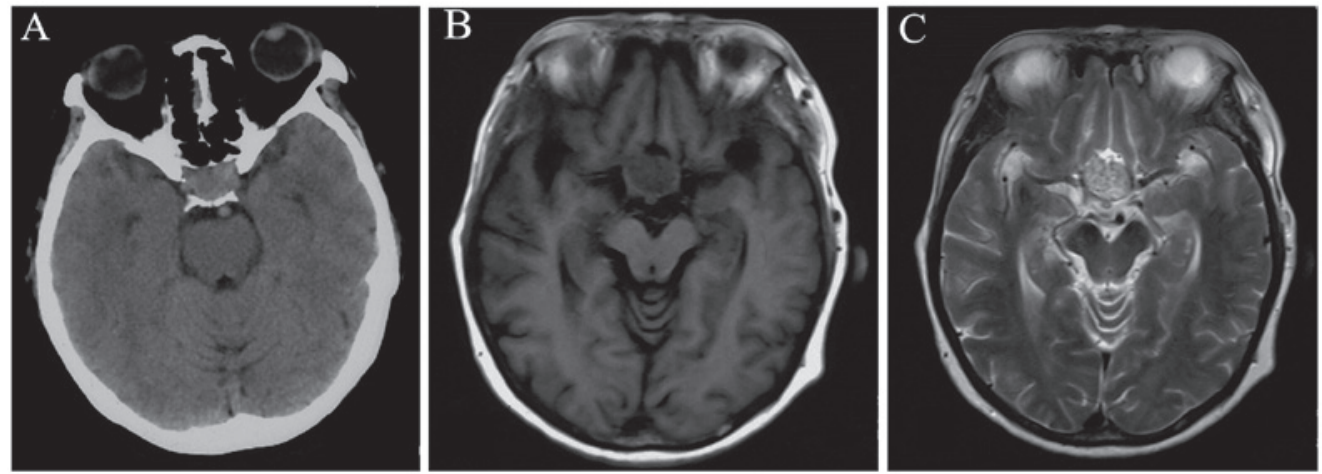

Figure 1. Representative computed tomography (CT) and magnetic resonance imaging (MRI) scans performed preoperatively. (A) Brain CT scan showing a sellar mass with heterogeneous hyperintensity. Brain MRI showing an intra- and suprasellar oval mass involving the hypothalamus and the foramen of Monro. The structures of the pituitary gland and pituitary stalk could not be clearly identified. The mass was (B) heterogeneously isointense on a representative T1WI and $(\mathrm{C})$ heterogeneously hyperintense on a representative $\mathrm{T} 2 \mathrm{WI}$
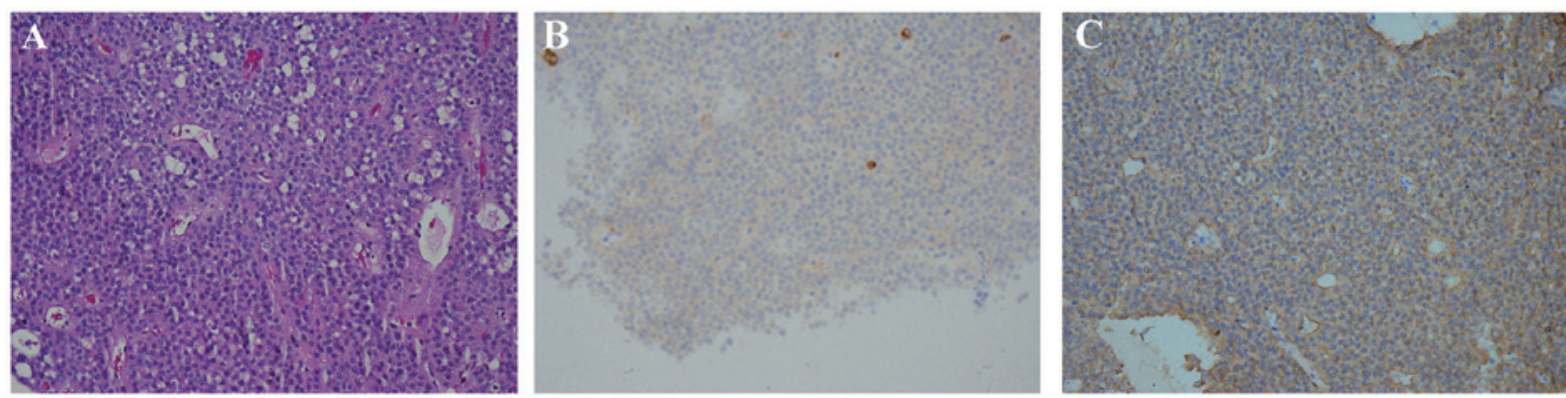

Figure 2. Representative images of histopathological and immunohistochemical staining of resected tumor tissue. (A) Histopathological examination confirmed the diagnosis of plurihormonal xanthogranulomatous pituitary adenoma, showing pituitary adenoma cells, cholesterol clefts, hemosiderin deposits, macrophages and foam cells (hematoxylin and eosin stain; original magnification, x100). Immunohistochemical staining showed the tumor was positive for (B) chromogranin A (magnification, x100) and (C) synaptophysin (magnification, x100).
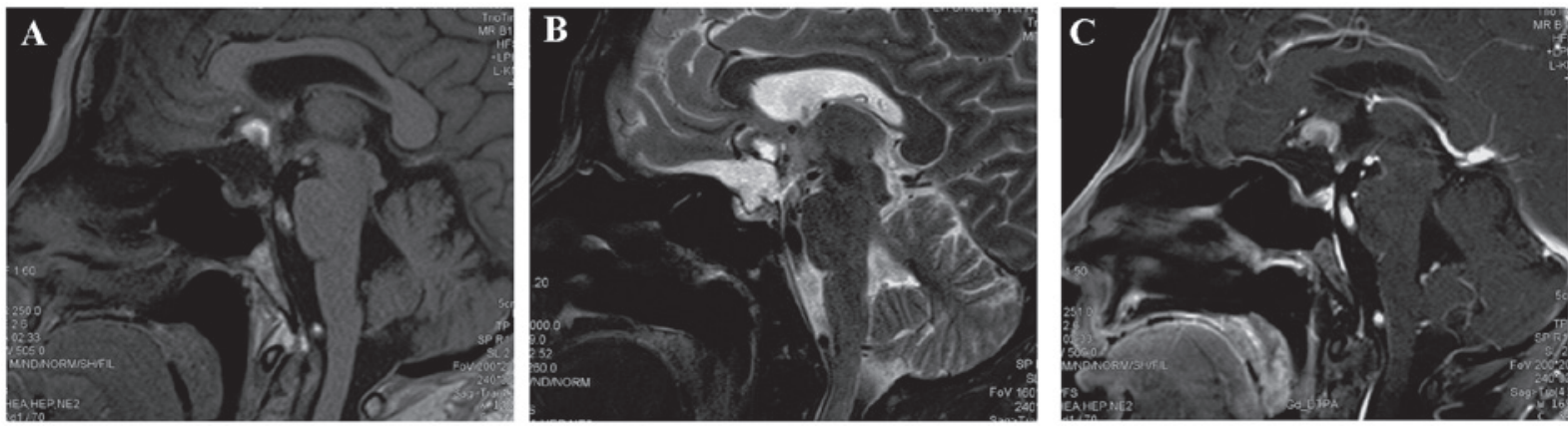

Figure 3. Postoperative magnetic resonance imaging confirmed partial tumor resection. The residual tumor above the optic chiasma exhibited (A) heterogeneous isointensity on a representative T1-weighted image and (B) heterogeneous hyperintensity on a representative T2-weighted image, with (C) significant contrast enhancement.

xanthogranulomas associated with pituitary adenoma. They identified 5 patients $(2.2 \%)$ with a remarkable xanthogranulomatous reaction among 231 consecutive cases of pituitary adenoma, and all 5 patients exhibited anterior pituitary insufficiencies (1). However, in the present case, the patient only presented with headache, vomiting and mild visual impairment, whereas there was no endocrine dysfunction or hypopituitarism. Thus, there may be no detectable connection between endocrine dysfunction and the size or extension of a pituitary adenoma. Sporadic cases have also manifested as obstructive hydrocephalus with acute changes in consciousness (19).
The imaging characteristics of xanthogranulomatous pituitary adenomas are non-specific. The tumors usually appear as isointense to hyperintense on T1WI and hypointense to hyperintense on T2WI, and contrast enhancement may be significant and heterogeneous $(1,4)$. In the present case, the sellar and suprasellar tumor extended into the third ventricle and exhibited heterogeneous isointensity on T1WI, with significant enhancement and heterogeneous hyperintensity on T2WI. The MRI characteristics may be associated with the multiple components of the tumor, with cholesterol clefts showing characteristic signals, with hyperintensity on T1WI 
and hypointensity on T2WI. Xanthogranulomatous pituitary adenomas may also exhibit atypical characteristics on MRI, including a dural tail, vascular encasement and intra-axial lesions in the posterior fossa (10).

The differential diagnosis of xanthogranulomatous pituitary adenoma is challenging and should include craniopharyngioma, Rathke's cleft cyst and granulomatous hypophysitis. Craniopharyngiomas most commonly occur in the suprasellar regions in young patients who present with visual impairment, intracranial hypertension and endocrine dysfunction. Moreover, calcifications are usually found on CT scans. Patients with Rathke's cleft cysts usually present with headache and endocrine dysfunction, and MRI of the tumors usually shows homogenous intensity with nodules in the cyst and circular enhancement. The main symptoms of granulomatous hypophysitis are hypopituitarism, diabetes insipidus, headache and visual impairment.

Accurate diagnosis of xanthogranulomatous pituitary adenoma is based on histopathological criteria. Microscopically, the typical histological characteristics of xanthogranuloma consist of cholesterol clefts, hemosiderin deposits, macrophages, chronic inflammatory infiltrates and fibrous proliferation. The histological findings in our patient were in accordance with the literature, supporting the diagnosis of xanthogranulomatous pituitary adenoma. Although xanthogranuloma may occur as an isolated entity, it usually coexists with other sellar tumors. In the present case, the presence of pituitary adenoma cells and the immunohistological staining results confirmed the diagnosis of associated plurihormonal pituitary adenoma. With regard to the differential diagnosis, enamel epithelial cells and cytokeratin may be found in adamantinomatous craniopharyngiomas; Rathke's cleft cysts usually display columnar epithelial cells, ciliated cells, hemorrhage and necrosis; and xanthogranulomatous hypophysitis mainly consists of foamy macrophages and small lymphocytes.

Xanthogranulomatous adenomas are benign, slow-growing entities. According to previous reports, surgical resection via the transsphenoidal approach is the preferred treatment, and gross total resection has been achieved in $73.3 \%$ of the cases $(1,6)$.In the present case, the tumor was resected via the right pterional approach; however, only the posterior portion of the tumor could be removed due to poor exposure. Previous reports indicated that headache and visual impairment may be completely relieved postoperatively, whereas endocrine dysfunction is difficult to treat. Our patient developed hypopituitarism and diabetes insipidus, which has not been previously reported. The specific pathogenic mechanisms responsible for these conditions remain unclear, and we hypothesized that these postoperative complications may be associated with dysfunction of the hypothalamus-pituitary axis due to inflammatory cell infiltration (20). Although recurrence was not observed in previously reported cases, close MRI follow-up is recommended given the rarity of xanthogranulomatous pituitary adenoma.

In conclusion, we herein reported a case of pituitary adenoma and concomitant xanthogranuloma in a female patient who developed diabetes insipidus postoperatively. Previously reported cases of this condition were reviewed, and definitive diagnosis of this condition relies on pathological examination. Xanthogranulomatous change should be carefully assessed in adenomas.

\section{Competing interests}

The authors declare that they have no competing interests.

\section{References}

1. Nishioka H, Shibuya M, Ohtsuka K, Ikeda Y and Haraoka J: Endocrinological and MRI features of pituitary adenomas with marked xanthogranulomatous reaction. Neuroradiology 52: 997-1002, 2010.

2. Paulus W, Honegger J, Keyvani K and Fahlbusch R: Xanthogranuloma of the sellar region: A clinicopathological entity different from adamantinomatous craniopharyngioma. Acta Neuropathol 97: 377-382, 1999.

3. Shirataki K, Okada S and Matsumoto S: Histopathological study of the 'cholesterol granuloma reaction' in the sellar and juxta-sellar tumors. No To Shinkei 40: 133-139, 1988 (In Japanese).

4. Yokoyama S, Goto M, Hirano H, Hirakawa W, Noguchi S, Hirahara K, Kadota K and Asakura T: Pituitary adenoma with cholesterol clefts. Endocr Pathol 9: 91-95, 1998.

5. Miyajima Y, Oka H, Utsuki S and Fujii K: Rathke's cleft cyst with xanthogranulomatous change-case report. Neurol Med Chir 51: 740-742, 2011.

6. Rahmani R, Sukumaran M, Donaldson AM, Akselrod O, Lavi E and Schwartz TH: Parasellar xanthogranulomas. J Neurosurg 122: 812-817, 2015.

7. Bao SS and Rapp R: Xanthogranuloma as an unsuspected cause of idiopathic central diabetes insipidus. Endocr Pract 20: e42-e46, 2014.

8. Ben Nsir A, Thai QA, Chaieb L and Jemel H: Calcified suprasellar xanthogranuloma presenting with primary amenorrhea in a 17-year-old girl: Case report and literature review. World Neurosurg 84: 866.e11-e14, 2015.

9. Kamoshima Y, Sawamura Y, Motegi H, Kubota K and Houkin K: Xanthogranuloma of the sellar region of children: Series of five cases and literature review. Neurol Med Chir (Tokyo) 51: 689-693, 2011.

10. Madan Mohan B, Mohamed E, Jain SK, Jain M and Jaiswal AK: Serial MR imaging in suprasellar xanthogranuloma: Growth pattern and new lesions. J Neuroimaging 25: 677-679, 2015.

11. Vajtai I, Kopniczky Z, Buza Z, Kovács J, Kovács Z, Varga Z, Bodosi $\mathrm{M}$ and Paulus W: Cholesterol granuloma at the sella region: A new method of the differential diagnosis of craniopharyngioma. Orv Hetil 142: 451-457, 2001 (In Hungarian).

12. Amano K, Kubo O, Komori T, Tanaka M, Kawamata T, Hori T and Okada Y: Clinicopathological features of sellar region xanthogranuloma: Correlation with Rathke's cleft cyst. Brain Tumor Pathol 30: 233-241, 2013

13. Burt MG, Morey AL, Turner JJ, Pell M, Sheehy JP and Ho KK: Xanthomatous pituitary lesions: A report of two cases and review of the literature. Pituitary 6: 161-168, 2003.

14. Gopal-Kothandapani JS, Bagga V, Wharton SB, Connolly DJ, Sinha S and Dimitri PJ: Xanthogranulomatous hypophysitis: A rare and often mistaken pituitary lesion. Endocrinol Diabetes Metab Case Rep 2015: 140089, 2015.

15. Yokoyama S, Sano T, Tajitsu K and Kusumoto K: Xanthogranulomatous hypophysitis mimicking a pituitary neoplasm. Endocr Pathol 15: 351-357, 2004.

16. Abla AA, Wilson DA, Eschbacher JM and Spetzler RF: Neurosurgical biopsy as the initial diagnosis of xanthogranuloma of the Erdheim-Chester disease variety of the infundibulum and optic apparatus: Letter to the editor. Acta Neurochir (Wien) 152: 925-927, 2010.

17. Reithmeier T, Trost HA, Wolf S, Stölzle A, Feiden W and Lumenta CB: Xanthogranuloma of the Erdheim-Chester type within the sellar region: Case report. Clin Neuropathol 21: 24-28, 2002.

18. Sulentić P, Cupić H, Cerina V and Vrkljan M: Xanthogranuloma of the sellar region in a patient with sarcoidosis. Acta Clin Croat 49: 61-65, 2010.

19. Liu ZH, Tzaan WC, Wu YY and Chen HC: Sellar xanthogranuloma manifesting as obstructive hydrocephalus. J Clin Neurosci 15: 929-933, 2008.

20. Sugata S, Hirano H, Yatsushiro K, Yunoue S, Nakamura K and Arita K: Xanthogranuloma in the suprasellar region. Neurol Med Chir 49: 124-127, 2009. 